\title{
VACUUM DRAINAGE IN THE THERAPY OF POSTOPERATIVE STERNO- MEDIASTINITIS IN CARDIAC SURGERY
}

\author{
Furgal A.A. ${ }^{1,4}$, Reva I.V. ${ }^{1,2}$, Reva G.V. ${ }^{1,2}$, Sorokin V.A. ${ }^{1,4}$, Garmash R.A. ${ }^{1}$, Garmash A.I. ${ }^{1}$, \\ Kupatadze D.D. ${ }^{3}$, Odintsova I.A. ${ }^{5}$, Verin V.K. ${ }^{6}$ \\ ${ }^{1}$ Far Eastern Federal University, Vladivostok, e-mail: RevaGal@yandex.ru; \\ ${ }^{2}$ International Medical Research and Education Center, Niigata, e-mail: avers2@yandex.ru; \\ ${ }^{3}$ St Petersburg State Pediatric Medical University, St. Petersburg, e-mail: ddkupatadze@gmail.com; \\ ${ }^{4}$ Pacific State Medical University, Vladivostok, e-mail: furgal86@bk.ru; \\ ${ }_{5}^{5}$.M. Kirov Military Medical Academy, St. Petersburg e-mail: odintsova.ia@yandex.ru; \\ ${ }^{6}$ North-Western State Medical University named after I.I. Mechnikov, St. Petersburg, e-mail: \\ vladimir.verin@spbmapo.ru
}

Aims.

The study is aimed at improvement of treatment outcomes of cardiosurgery patients and assessment of the efficacy of vacuum-assisted closure (VAC) therapy.

Materials and methods.

The study included 57 cardiosurgery patients after sternotomy, whose postoperative course was complicated by sterno-mediastinitis. The patients were treated in the period of 2012-2018. The patients were subdivided into the following clinical groups: 1) alternating vacuum suction (13 persons), 2) constant vacuum suction (14 persons), and 3) open management (30 persons).

Results.

Vacuum drainage in the alternating mode induced regenerative processes in the area of the wound surface to a greater extent in comparison with the groups of constant vacuum drainage and open management method, and enabled an increase in the number of fibres in the cicatricial tissue and their proper and orientation according to the dominant direction of load, in a shorter time period. An advantage of the vacuum drainage effect on the wound process, as compared to the open management, was more pronounced stimulation of growth and improvement in quality of granulations. Through directional deformation of tissues, the vacuum bandage reduces the extent of wound surface and wound size, therefore additional mobilisation of local tissue prior to secondary wound closure was not required. Application of vacuum drainage on the wound made it possible to eliminate the excess wound exudate, thus stimulating angiogenesis. In all study groups, longer periods of wound healing were observed in patients with concomitant diabetes mellitus type 2.

Conclusions.

Vacuum drainage in the alternating mode showed the best results in the treatment of postoperative sternomediastinitis in cardiac surgery practice.

Keywords: cardiac surgery, alternating vacuum, regeneration, granulation, mechanisms of angiogenesis, growth factors, mesenchymal stem cells, pericytes, circulating progenitor cells.

\section{INTRODUCTION.}

Despite the advances in surgery, antibacterial therapy, and preventive medicine, postoperative sternomediastinal infections do still affect the morbidity and fatality rates, the duration of hospital stay, and the costs of treating a cardiac surgery patient.

Postoperative complications in cardiac surgery in general, and infectious complications of sternotomy wounds in particular, are an increasingly relevant problem, as there are ever more geriatric and diabetes mellitus patients $[1 ; 2]$.

Despite the long history of median sternotomy, this approach has a number of drawbacks, including the infectious complications at the site of surgery. Such complications result in a surficial 
or deep wound infection, diastasis and fragmentation of the sternum, and destabilization of the thoracic bone frame [3; 4].

Even when adjusted for state-of-the-art treatment and prevention methods, postoperative mediastinitis occurs in $1.1 \%$ to $19 \%$ of cardiac surgeries [5;6]. Suture failure and sternotomy wound infection is a life-threatening complication with fatality rates of $14 \%$ to $75 \%$ in the risk group [7]. Anterior sterno-mediastinitis (ASM) is associated with a considerable reduction in longterm survival $[8 ; 9]$. Risnes et al. have shown that the survivors of ASM have a 59\% higher risk of dying within 10 years after surgery compared to non-ASM patients [10; 11]. This is because the mediastinitis-associated chronic inflammatory process may adversely affect thrombogenicity and the shunt patency, resulting in lower long-term survival [12].

ASM is a very costly complication. Treating an ASM patient is 2 to 3 times more expensive on average than treating a similar non-ASM patient [13; 14].

The goal hereof is to improve cardiac surgery outcomes and to analyze the effectiveness of VAC therapy. This goal logically led to an objective of assessing the clinical and morphological parameters, the induction of regenerative processes of postoperative wounds in cardiac surgery patients subjected to open treatment, variable or constant vacuuming.

MATERIALS AND METHODS. Patients were examined and treated at four Russian cardiac surgery centers:

1. Cardiovascular Surgery Center of the Far-Eastern Federal University Health Center, Vladivostok.

2. Cardiac Surgery Unit of the Primorye Regional Clinical Hospital No. 1, Vladivostok.

3. Federal Cardiovascular Surgery Center of the Ministry of Health, Khabarovsk.

4. A.N. Bakulev National Medical Research Center of Cardiovascular Surgery.

Group 1 comprised patients subjected to variable vacuum drainage; Group 2 had constant vacuum drainage; Group 3 had open treatment. Experts involved in the analysis and interpretation of research-related histological data were not given any details on how the patients were grouped so as to reduce the systematic statistical error rate and to maximize the objectivity of histological interpretation.

The study followed the requirements of the Russian Ministry of Health and Medical Industry No. 82 of April 29, 1994 and used the nomenclature of clinical laboratory studies of the Ministry of Health (Order 64 of February 21, 2000) pursuant to the Helsinki Declaration (2000). Prospective controlled comparative studies monitored the morphological changes that occurred as part of the regeneration of infected sternotomy wounds in full compliance with the principles of evidence-based medicine. The research team used clinical and classical morphological methods to monitor the reparative processes in postoperative sterno-mediastinitis patients to further analyze the obtained data statistically. The research followed the guidelines by Vishnevsky et al. (2005), who interpreted "postoperative sterno-mediastinitis" as an umbrella term; due consideration was given to 
the mediastinitis classifications by Ivanov (1959), as well as to Abakumov's Classification of Acute Mediastinitis (2010), as sternal infections nearly always affect not only the subcutaneous fat, but also the ribs, the cartilage, and the muscles of the thorax, clavicles, and anterior mediastinum. This research used negative pressures (NP) of $-50 \mathrm{mmHg}(\mathrm{min})$ to $-125 \mathrm{mmHg}(\mathrm{max}$ ), as the use of NP in the lower and medium therapeutic range is the most effective option. A Suprasorb CNP P1 aspirator was used for variable vacuum therapy. For constant VAC therapy, pressure was set to 120-170 mmHg using a Medela Vario-18 ac/dc.

Open dressings were performed 2 to 5 times a day. The exact number of dressings depended on the wound exudation rate. The bandages were impregnated with a combined antimicrobial topical drug (chloramphenicol + methyluracil). Regardless of the treatment method, the wound was sanitized with chlorhexidine and betadine iodine solutions when dressed. The study was permitted by the Ethics Committee of the Pacific State Medical University and Far-Eastern Federal University. The Table below shows how the patients were grouped.

Patient breakdown by postoperative treatment methods

\begin{tabular}{|c|c|c|}
\hline Group No. & $\begin{array}{c}\text { Postoperative treatment } \\
\text { methods }\end{array}$ & \begin{tabular}{c} 
Number of patients \\
\hline 1
\end{tabular} \\
\hline 2 & Variable vacuum & 13 \\
\hline 3 & Constant vacuum & 30 \\
\hline Total: & Open treatment & 57 \\
\hline
\end{tabular}

The research team used morphological tests to objectively evaluate the regeneration of damaged tissue so as to address the possible mechanisms of reparative regeneration as affected by open treatment with constant or variable vacuum applied. Tissue was sampled for comparative tests on the day ASM was diagnosed and then every four days after diagnosing and starting to treat ASM; samples were also taken when sanitizing the post-sternotomy wounds surgically as indicated. Sampled tissue volume did not exceed $1 \mathrm{~mm}^{3}$.

RESULTS. Sections of tissue were made from the biopsy samples for classical morphological testing and further hematoxylin and eosin staining. The preparations were rich in fat and blood vessels with lavers of lnne fihrous connective tissue, see Figure 1.
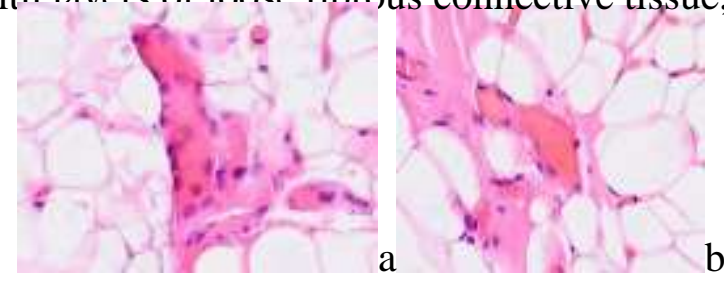


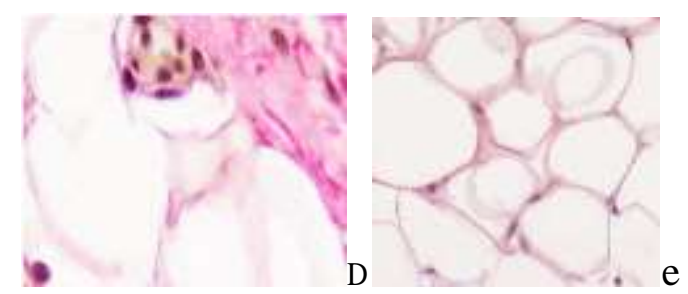

Figure 1. Biopsy samples taken from the postoperative wound during surgery. The patient had a normal body weight and no diabetes mellitus (a-e). Hematoxylin and eosin staining.

Microphotograph. x200 zoom

Biopsy samples of the wound surface in Type 2 diabetes patients. Sampled after open wound treatment, see Figures 2 and 3.
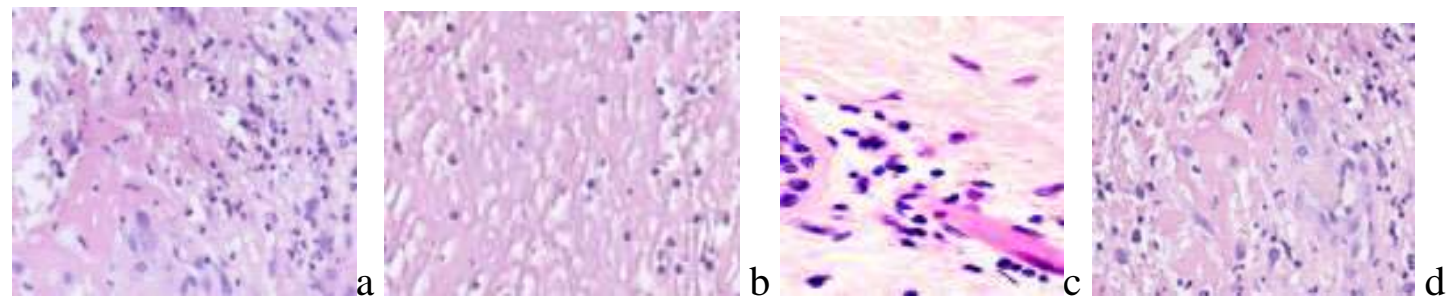

Figure 2. Leukocyte necrotic surface layer; leukocyte infiltration of the wound in a prepared biopsy sample of the wound surface in Type 2 diabetes patients after (a) open wound treatment; (b) variable vacuum drainage; (c),(d) constant vacuum drainage. Hematoxylin and eosin staining. Microphotograph. Zoom: $(a),(b),(d) \times 200 ;(c) \times 400$
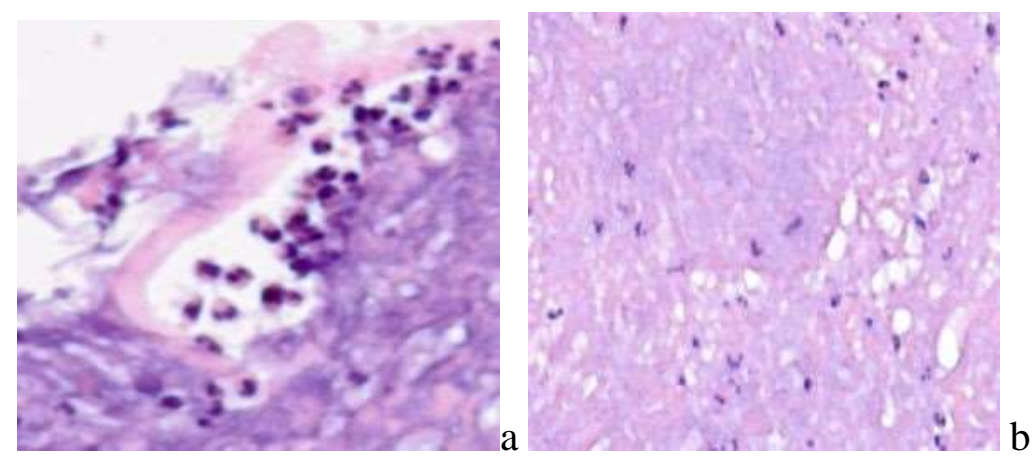

Figure 3. Vascular granulation layer. Leukocyte infiltration outside the vessel in a prepared wound biopsy sample taken from a Type 2 diabetes patient after variable vacuum treatment. Hematoxylin and eosin staining. Microphotograph. x200 zoom.

Hyperemia-affected vessels (a), tissue infiltration $(b)$ detected

The key feature of this stage of wound healing is that the edges are patched together by granulations rather than a scar. An inflammatory response supersedes the edema and melts the dead tissue to clean the wound; as it is over, granulations slowly emerge to gradually fill the defect.

Testing the micropreparations revealed that variable VAC therapy had resulted in the least 
hyperemia and edema at Stage I in all patients groups with various pathologies, see Figure 4.
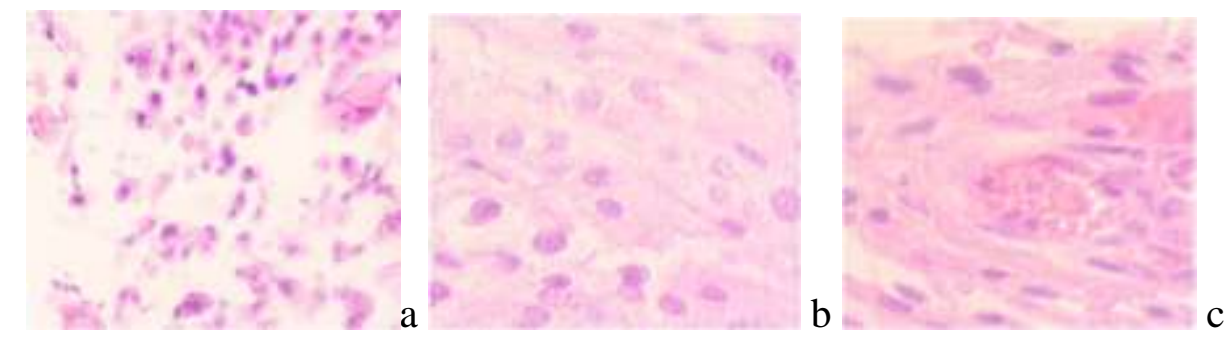

Figure 4. Blood vessels in the prepared biopsy sample of the wound surface taken from non-DM, non-obese patients on Day 4 after diagnosing ASM and sanitizing the post-sternotomy wound surgically. The patients underwent: (a) open treatment; $(b)$ variable vacuum drainage; $(c)$ constant vacuum drainage. Hematoxylin and eosin staining. Microphotograph.

$$
\text { x200 zoom }
$$

Granulations look differently depending on how far they have progressed. Normal granulations initially look like fine-grained tissue covered with cloudy gray-greenish plaque; the tissue is juicy and rich in thin-walled vessels, making such granulations easy to bleed. Older granulations are paler and denser; they are no longer grained, instead of turning into whitish, dense scars.

A granulation comprises six interfacing layers: a leukocyte necrotic surface layer; a surface layer of vascular slings; a layer of vertical vessels; a maturing layer; a layer of horizontal fibroblasts; and a fibrous layer.

Monitoring the open-treated patients revealed granulations emerging on the boundaries between wound edges on Day 4. Those consisted of multiple close-spaced granules. The granules consisted of amorphous substances, sling-shaped vascular capillaries, histiocytes, fibroblasts, polyblasts, lymphocytes, multinuclear wandering cells, collagen and elastic fibers, as well as segmented leukocytes. Variable-vacuumed patients had earlier granulations, as pinkish-red nodules having a size of a millet grain were detected on the blood- and necrosis-free sites in only two days. There were many more granules on Day 3, with newly formed granulations covering the wound surface on Day 4 and 5. Strong and healthy granulations of pinkish-red color did not bleed, were uniformly grainy and very dense, emitting only a small amount of cloudy purulent exudate. The exudate contained numerous dead cells of the local tissue, pus corpuscles, erythrocyte contaminations, segmented leukocytes, and various microflora with its metabolic byproducts. Reticuloendothelial cells and white blood cells emigrated to this exudate; vascular capillaries and fibroblasts grew into it. Preparations contained granulations of multiple small plethoric blood vessels (capillaries and arterioles) within multiple proliferating cellular elements in-between: histiocytes, lymphocytes, plasma cells, epithelioid cells, and fibroblasts. In all cases, variable vacuum drainage was associated with more active angiogenesis, see Figure 5. 


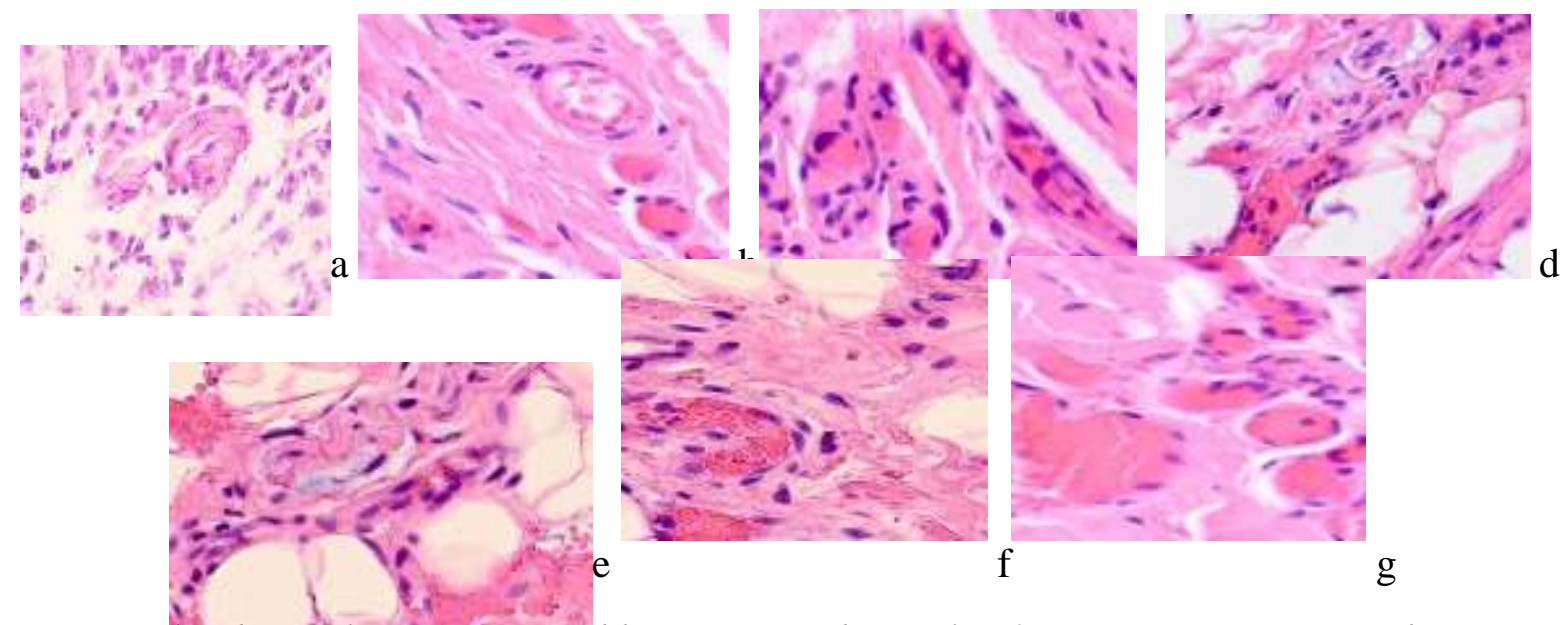

Figure 5. Blood vessels in a prepared biopsy wound sample of COPD patients treated (a) openly;

(b) to $(f)$ by variable vacuum drainage; $(g)$ by constant vacuum drainage.

Hematoxylin and eosin staining. Microphotograph. x200 zoom

Exposing the wound surface to variable vacuum drainage was noted to cause earlier granulations, lesser vascularization, the wound surface to be filled with maturing connective tissue, and earlier epithelization of the wound surface, see Figure $5 \mathrm{~b}$ to $5 \mathrm{f}$.

In the second reparation period, the newly formed granulations between the wound edges matured rapidly, and collagen was generated at a high rate. On the one hand, this reduced the amount of vessels and cellular elements; on the other hand, it also increased the number of collagen and elastic fibers, see Figure 6.
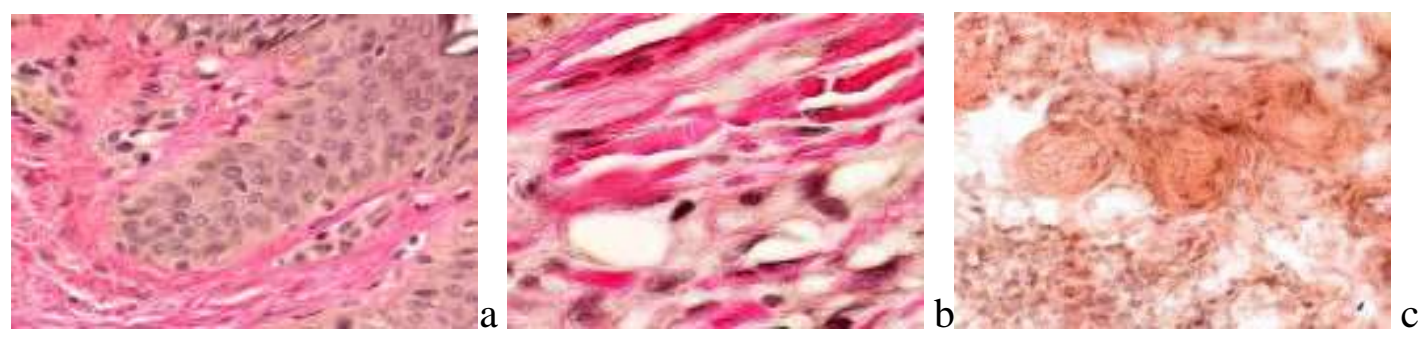

Figure 6. Blood vessels in a prepared biopsy wound sample of CKF patients treated (a) openly; (b) by variable vacuum drainage; (c) by constant vacuum drainage. Van Gieson staining.

Microphotograph. x200 zoom

The third reparation stage featured active connective tissue growth, whereby a strong scar was formed. There were significantly more fibrous structures in the scar, and their bundles became codirectional with the vector of the prevalent load. Accordingly, the vascular and cellular element populations dropped significantly, see Figure 7.
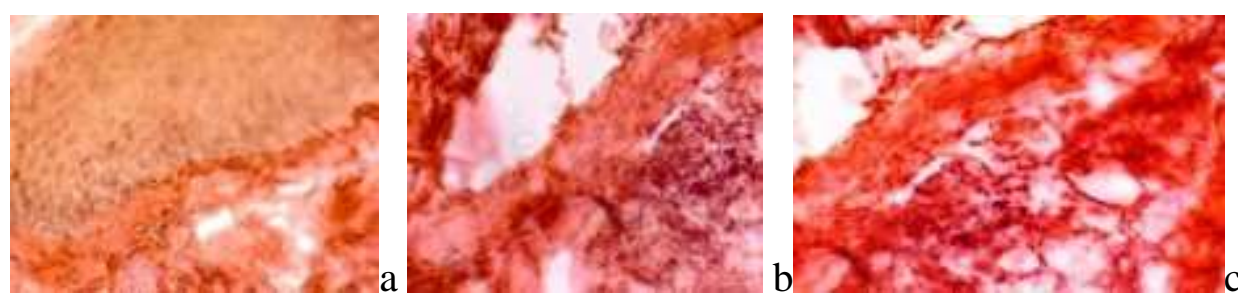

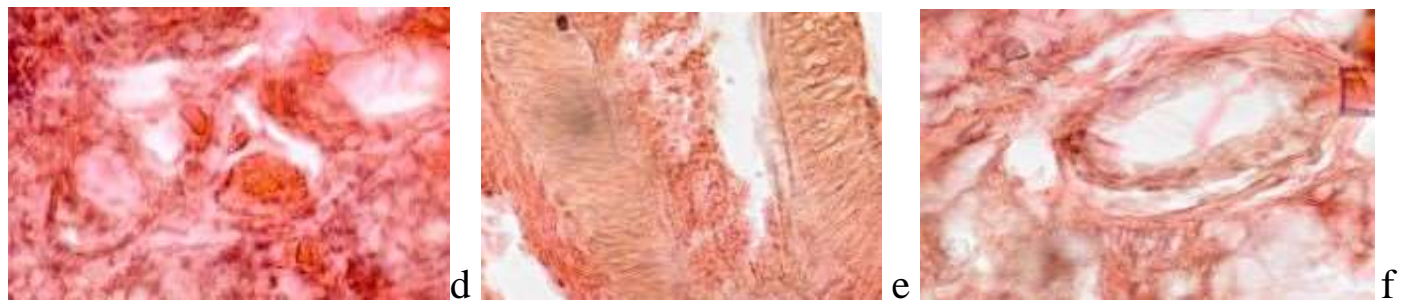

Figure 7. Prepared biopsy sample of the wound surface from a CKD patient, taken 25 days after diagnosing ASM: (a) open-treated; $(b)$ to $(d)$ variable-vacuumed; $(e),(f)$ constant-vacuumed. Van Gieson staining. Microphotograph. x200 zoom

As the scar tissue restructured and the fibers acquired a more longitudinal orientation, the population of cellular elements dropped, while single small vessels remained. Vacuum-drained patients had the most properly-directed fibers in their scar tissue, see Figure 8.
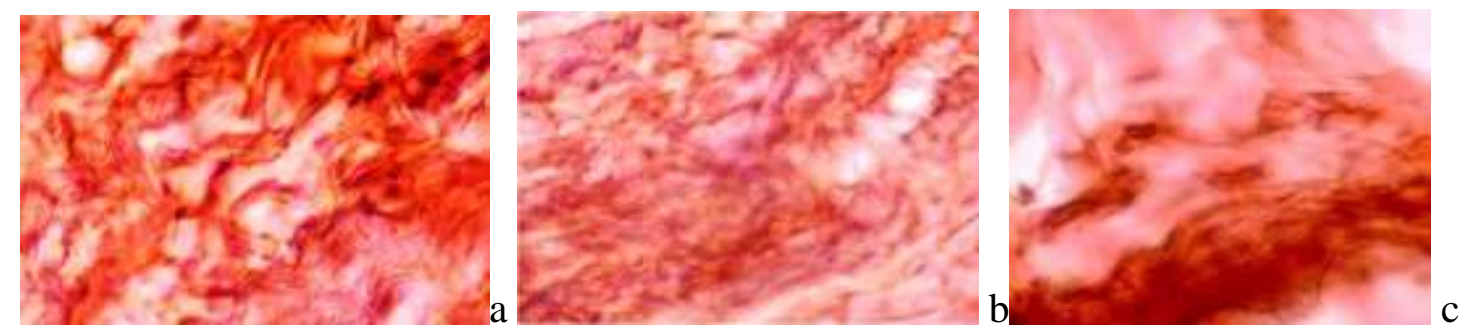

Figure 8. Connective-tissue scarring in obese patients on Day 45: (a) variable-vacuumed; (b), (c) constant-vacuumed. Van Gieson staining. Microphotograph. x200 zoom

Histological testing showed that vacuum drainage improved wound healing by:

1) reducing the local edema;

2) boosting the local tissue perfusion;

3) reducing the microbial contamination of the wound;

4) configurably deforming the wound bed and reducing the wound cavity volume;

5) reducing the wound exudation;

6) maintaining the optimal humidity of the wound environment, which was necessary for the wound to heal properly.

Clinical and morphological monitoring showed that open-treated and constant-vacuumed Type 2 diabetes mellitus patients had (compared to their variable-vacuumed counterparts): longer edema and infiltration of the wound-adjacent tissue; far slower granulation accompanied by capillary and arteriole growth; far slower emptying of the vessels; far slower depopulation of connective-tissue cells and bloodstream migrants.

CONCLUSIONS. Variable vacuum drainage induced wound regeneration to a greater extent than constant vacuum drainage or open treatment; scar fiber growth and load-appropriate direction were attained faster. Compared to open treatment, vacuum drainage better stimulates the 
growth of granulations and improves their quality. Since a vacuum bandage deforms tissue in a specific direction, it reduces the wound surface and size so that mobilizing more of the local tissue before secondary wound closure might become unnecessary. Vacuum wound drainage removes excess exudate and stimulates angiogenesis.

FINANCIAL SUPPORT AND SPONSORSHIP

The work was carried out with the support of the FEFU Scientific Foundation, State Task 17.5740/2017/6.7.

\section{CONFLICTS OF INTEREST}

The authors declare no conflict of interest

\section{SUPPLEMENTARY DATA (DOI)}

\section{REFERENCES}

1. Uskela S., Kärkkäinen J.M., Eränen J. et al. Percutaneous coronary intervention with drug-coated balloon-only strategy in stable coronary arterydisease and in acute coronary syndromes: An allcomers registry study. Catheterization and Cardiovascular Interventions, 2018 vol. 93, no 5, pp 893-900, doi 10.1002/ccd.27950.

2. Kaláb M., Karkoška J., Kamínek M. et al. Reconstruction of massive post-sternotomy defects with allogeneic bone graft: four-year results and experience using the method. Interactive CardioVascular and Thoracic Surgery, 2016. vol. 22, no 3, pp. 305-313, doi 10.1093/icvts/ivv322.

3. Kaláb M., Karkoška J., Kamínek M. et al. Successful three-year outcome in a patient with allogenous sternal bone graft in the treatment of massive post-sternotomy defects. International Journal of Surgery Case Reports, 2015, vol. 7, pp. 6-9, doi 10.1016/j.ijscr.2014.12.027.

4. Prifti E., Demiraj A., Xhaxho R. Modified Port-Access Technique for the Treatment of Aortic Dissection after Previous. Cardiac Surgery. Texas Heart Institute Journal, 2017, vol. 44, no 3, pp. 202-204, doi 10.14503/thij-16-5800.

5. Kirmani B.H., Jones S.G., Malaisrie S.C. et al. Limited versus full sternotomy for aortic valve replacement. Cochrane Database of Systematic Reviews, 2017. vol 10, no 4, p. 4, doi 10.1002/14651858.cd011793.pub2.

6. Suzuki K., Ishigami N., Akuzawa S. Treatment of Mediastinitis after Open Heart Surgery Using Negative Pressure Wound Therapywith Irrigation. Kyobu Geka, 2018, vol. .71, no 11, pp. 916-918.

7. Onan I.S., Yildiz O., Tüzün B. et al. Vacuum-Assisted Closure for Mediastinitis in Pediatric Cardiac Surgery: A Single-Center Experience. Artificial Organs, 2019, vol. 43, no 2, pp. 119-124, doi 10.1111/aor.13321.

8. Copeland H., Newcombe J., Yamin F. et al. Role of Negative Pressure Wound Care and Hyperbaric Oxygen Therapy for Sternal Wound Infections After Pediatric Cardiac Surgery. World Journal for Pediatric and Congenital Heart Surgery, 2018, vol. 9, no 4, pp. 440-445, doi 10.1177/2150135118772494. 
9. Lentini S., Monaco F., Tancredi T. et al. Surgical treatment of pericardial cyst through median sternotomy. Minerva chirurgica. 2009, vol. 64, no 1, pp. 105-109.

10. Buja A., Zampieron A., Cavalet S., et al. An update review on risk factors and scales for prediction of deep sternal wound infections. International Wound Journal, 2012, vol. 9, no 4, pp. 372-386, doi 10.1111/j.1742-481x.2011.00896.x.

11. Hulman M., Bezak B., Artemiou P. et al. Wound infections after median sternotomy treated by VAC therapy, summary of results, and risk factor analysis. Bratislava Medical Journal, 2018, vol. 118, no 12, pp. 736-739, doi 10.4149/bll_2017_139.

12. Armaganidis A., Diplas D., Floros I. et al. Effect of median sternotomy on respiratory system compliance in humans: evaluation without sophisticated instrumentation. Interactive CardioVascular and Thoracic Surger, 2009, vol. 8, no 1, pp. 22-26, doi 10.1510/icvts.2008.182261. 13. Suzuki R., Mikamo A., Tanaka Y et al. Apico-aortic valved conduit for aortic stenosis in patients who could not tolerate mediansternotomy. Kyobu Geka, 2013, vol. 66, no 13, pp. 11321136.

14. Podestà A., Parodi E., Dottori V. et al. Minimally invasive thoracotomy for myocardial revascularisation. A study of 32 cases. Minerva cardioangiologica, 2002, vol. 50, no 2, pp. 161166. 